\title{
Masking model for accurate colorimetric characterization of LCD
}

\author{
Nobuhiko Tamura \\ Norimichi Tsumura \\ Yoichi Miyake
}

\begin{abstract}
A color management system (CMS) such as ICC profile or sRGB space have been proposed for color transformation and reproduction of cross media. In such a CMS, accurate colorimetric characterization of a display device plays a critical role in achieving device-independent color reproduction. In the case of a CRT, colorimetric characterization based on a GOG model is accurate enough for this purpose. However, there is no effective counterpart in liquid-crystal displays (LCDs) since the characterization of an LCD has many difficulties, such as channel interaction and non-constancy of channel chromaticity. In this paper, a new method of display characterization is proposed which is applicable to the assessment of color reproduction of LCDs. The proposed method characterizes an electro-optical transfer function considering both channel interaction and non-constancy of channel chromaticity. Experimental results show that the proposed method is very effective in the colorimetry of LCDs.
\end{abstract}

Keywords - Display characterization, LCDs, masking model, channel interaction.

\section{Introduction}

Flat-panel displays have become increasingly popular because of its low power consumption and versatility with respect to placement. Therefore, it is important to establish an accurate CMS in LCDs. In CMS, one of the most important characteristics of the color reproduction is the relationship between digital input values RGB and XYZ tristimulus values of display. If the relationship is known, we can handle the color on LCDs based on device-independent XYZ tristimulus values. The GOG model ${ }^{1}$ is a well-known characterization model for CRT displays. XYZ values of an arbitrary digital input can be predicted accurately by using the GOG model. However, characterization of LCDs is rather difficult compared to CRT displays with the presence of channel interaction and non-constancy of channel chromaticity. Many researches have done characterizing colorimetry of display devices. The S-curve model ${ }^{2,9}$ is proposed for LCD colorimetry, which characterizes the electro-optical transfer function using an S-shaped curve. The polynomial model and matrix model ${ }^{3}$ are also proposed for the characterization of display devices. However, the accuracy of these conventional models is not high enough for the characterization of LCDs because of difficulties such as channel interaction and the non-constancy of chromaticity. The required accuracy of the characterization model is essentially different by its usage. In this paper, we aimed at an accuracy which is indistinguishable by color professionals from a color-difference point of view.

In this paper, two models are proposed for the characterization of LCDs: the Masking model and the Modified Masking model. Proposed models can be classified as the model that does not consider the internal structures of displays. The relationship between the digital input and luminance of each channel were approximated using spline interpolation. The proposed models also took into consideration two major problems in characterizing LCD colorimetry; channel interaction and non-constancy of channel chromaticity. In addition to the measurement of RGB primary colors, C, M, Y, Gr (Cyan, Magenta, Yellow, and Gray), secondary and tersiary colors were measured to approximate the color variation caused by channel interaction. For the Masking model, the primary color vector was calculated using principal component analysis (PCA) to minimize the error caused by variation in channel chromaticity. On the other hand, the Modified Masking model further considers a higher-order principal component to characterize LCDs, where channel chromaticity is highly dependent on the digital inputs. A comparison with conventional characterization methods using three LCDs proved that the proposed method is more effective for colorimetric characterization of LCDs.

\section{Review of conventional display charac- terization models}

\subsection{GOG, S-curve, and polynomial models}

The GOG, polynomial, and S-curve models have the same structure as defined in Eq. (1).

$$
\left[\begin{array}{c}
X \\
Y \\
Z
\end{array}\right]=\left[\begin{array}{lll}
X_{R, \text { max }} & X_{G, \text { max }} & X_{B, \max } \\
Y_{R, \text { max }} & Y_{G, \max } & Y_{B, \max } \\
Z_{R, \max } & Z_{G, \max } & Z_{B, \max }
\end{array}\right]\left[\begin{array}{l}
R\left(d_{R}\right) \\
G\left(d_{G}\right) \\
R\left(d_{B}\right)
\end{array}\right]+\left[\begin{array}{c}
X_{0} \\
Y_{0} \\
Z_{0}
\end{array}\right],
$$

where $\mathrm{X}_{0}, \mathrm{Y}_{0}, \mathrm{Z}_{0}$ are the $\mathrm{XYZ}$ of the ambient flare and $\mathrm{X}_{\mathrm{i} \text {,max }}$, $Y_{i, \text { max }}, Z_{i, \text { max }}(i=R, G, B)$ are the maximum XYZ of each channel after black correction. $\mathrm{R}\left(\mathrm{d}_{\mathrm{R}}\right)$ for each model was defined as follows: 
GOG model:

$$
R\left(d_{R}\right)=\left\{\begin{array}{cl}
\left\{k_{g, R} d_{R}+k_{o, R}\right\}^{\gamma_{R}} & ,\left\{k_{g, R} d_{R}+k_{o, R}\right\} \geq 0 \\
0, & \left\{k_{g, R} d_{R}+k_{o, R}\right\}<0
\end{array}\right.
$$

Polynomial model:

$$
R\left(d_{R}\right)=a_{R} d_{R}^{2}+b_{R} d_{R}+c_{R}
$$

S-curve model:

$$
R\left(d_{R}\right)=A_{R R} f\left(d_{R}\right)+A_{R G} f^{\prime}\left(d_{G}\right)+A_{R B} f^{\prime}\left(d_{B}\right),
$$

where $f\left(d_{R}\right)=d_{R}^{\alpha} /\left(d_{R} \beta+C\right)$ and $f^{\prime}$ is the derivative of $f$.

All variables except $d_{R}, d_{G}$, and $d_{B}$ are constants, which were calculated to minimize the error between training XYZ and predicted XYZ. $G\left(\mathrm{~d}_{\mathrm{G}}\right)$ and $\mathrm{B}\left(\mathrm{d}_{\mathrm{B}}\right)$ were similarly defined. The GOG model characterizes each transfer step from digital input to XYZ of the CRT through video card, gun, and phosphor gamma. The prediction of XYZ using the GOG model is accurate enough for CRTs. However, it is not always effective when the GOG model is applied to LCDs because of structural difference between CRTs and LCDs. The S-curve model has the same structure as the GOG model but differs in the non-linear relationship between the digital input and luminance of each channel. The S-curve model characterizes the relationship using the S-shaped function which is defined in Eq. (4) instead of the gammashaped function used in the GOG model. The S-curve model further considers the non-chromaticity constancy of LCDs. However, the S-curve model requires a great deal of training data compared to other models because its large number of unknown coefficients. Some manufacturers transform the S-curve characteristic into the gamma characteristic on an integrated circuit (IC). Figure 1 shows the LCD's luminance curve for the $\mathrm{R}$ channel (iiyama 4635U) which looks like a gamma-shaped curve rather than an S-shaped curve. The GOG, S-curve, and polynomial models always have a prediction error due to the channel interaction because Eq. (1) assumes the channel additivity.

\subsection{LUT method}

Characterization using the LUT method requires a considerable amount of measurement. The difficulty with the LUT method is not only the measurement time required to construct the LUT, but the amount of data needed to describe the characteristics of the display. For instance, the inclusion of the LUT in the ICC profile would increase the overhead. Unless the system far deviates from linearity, such as the additivity and constancy of channel chromaticity, the LUT method would be of little value.

\subsection{Matrix model}

The matrix model is defined in Eq. (5). Firstly, the non-linear relationship between the digital input $\mathrm{d}_{\mathrm{R}}$ and the lumi-

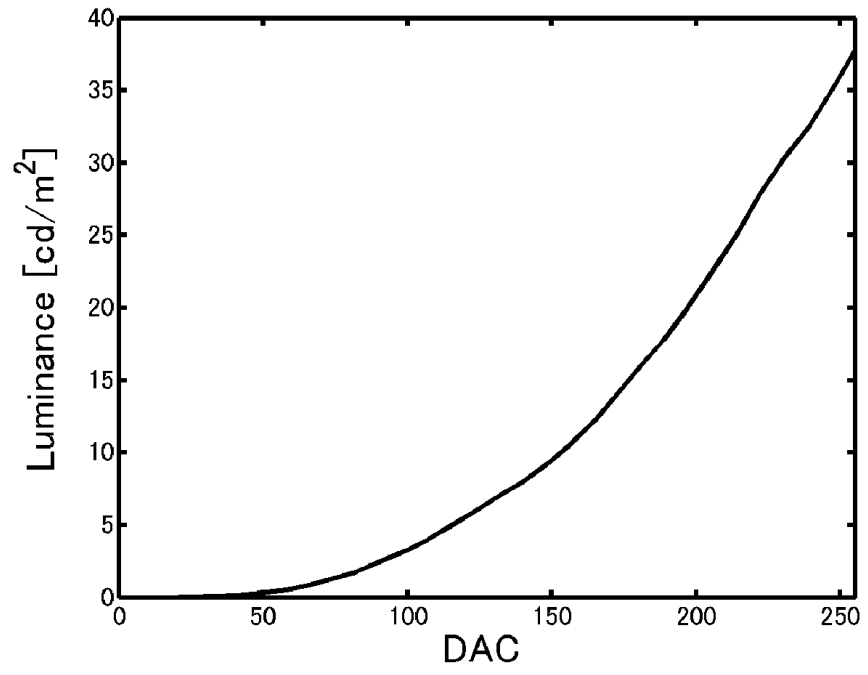

FIGURE 1 - Gamma-shaped electro-optical transfer function of an LCD (iiyama AS4635U).

nance $\mathrm{R}$ was calculated using the measured data. Secondly, matrix A was calculated to minimize the error using 32 neutral colors (see Refs. 3 and 9). Matrix model can handle the channel interaction by including cross-terms RG, GB, BR, as RGB as regression variables. However, the relationship between input values and the effect of channel interaction is not quite so simple (see Ref. 8) that the accuracy of the characterization by this method is high enough:

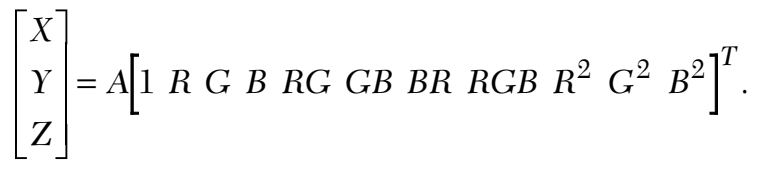

\section{Masking model \\ 3.1 Channel interaction}

Figure 2 shows two electro-optical transfer functions for the LCD (SHARP LL-T180) for the R channel where the digi-

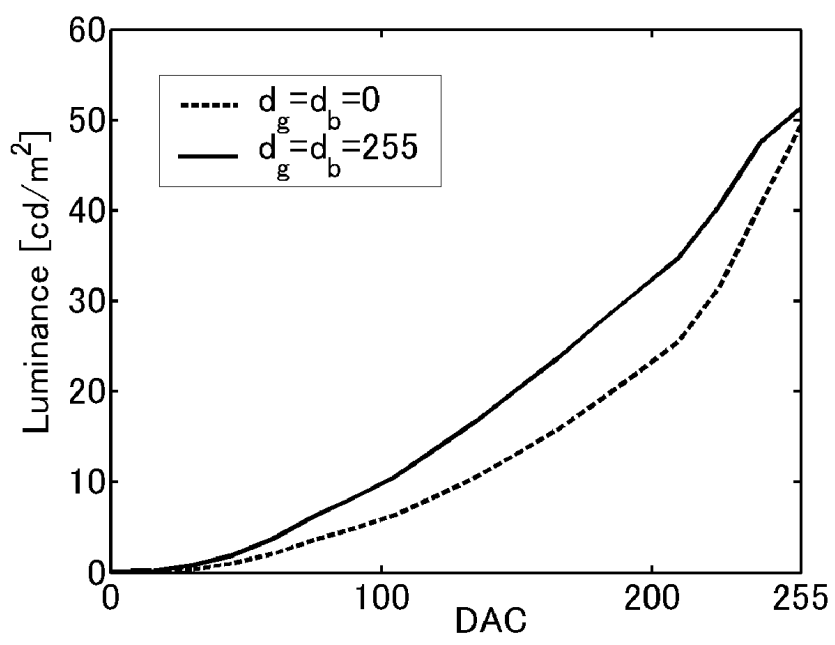

FIGURE 2 - Difference of Electro-optical transfer function caused by channel interaction. 


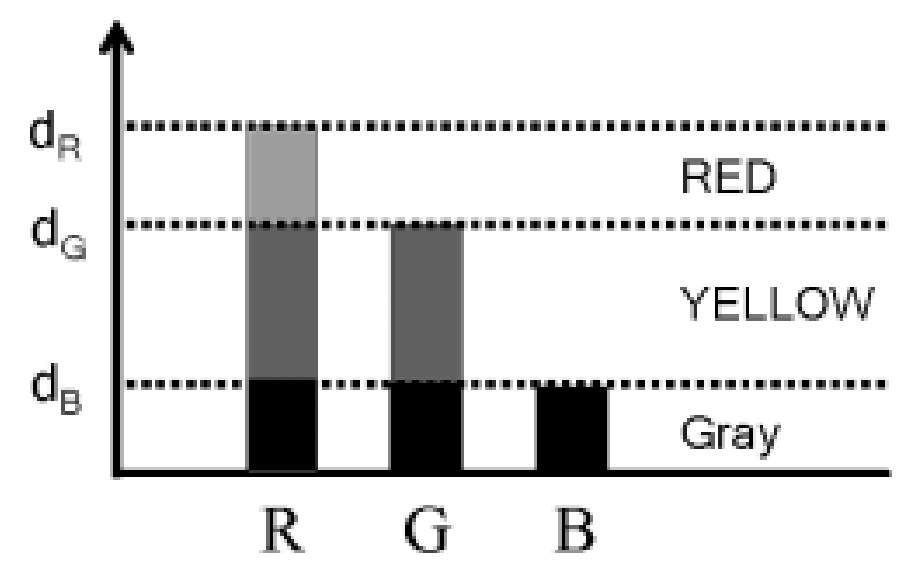

FIGURE 3 - Masking in the proposed model.

tal input of G, B channels are kept at 0 and 255 respectively. First, based on Eq. (1), $\mathrm{R}\left(\mathrm{d}_{\mathrm{R}}\right)$ for each case was calculated. Secondly, $\mathrm{Y}_{\mathrm{R} \text {,max }}$ was multiplied by $\mathrm{R}\left(\mathrm{d}_{\mathrm{R}}\right)$ to determine the luminance of the $\mathrm{R}$ channel. Note that Fig. 2 shows the luminance of the $\mathrm{R}$ channel, not the total luminance of the display. The presence of channel interaction can be seen in Fig. 2. Although it is known that the channel interaction is caused by capacitive coupling, formulation is rather difficult since the effect of interconnection largely depends on the internal structure of the driving circuit. In the Masking model, we directly measured the secondary and tersiary color to approximate the channel interaction. Then, the XYZ of the arbitrary digital input was calculated using R, G, B, C, M, Y, Gr characteristics. The concept of the Masking model is similar to that of the UCR (under color removal) in printing technology. The same amount of each RGB digital count is replaced by gray for which the digital count is equal to the smallest digital counts in RGB (see Fig. 3). Similarly, the remaining digital counts are replaced by red and yellow as shown in Fig. 3.

Let $\mathbf{I}\left(d_{R}, d_{G}, d_{R}\right)$ be the XYZ value of the display in vector form, which corresponds to digital input $\left(\mathrm{d}_{\mathrm{R}}, \mathrm{d}_{\mathrm{G}}, \mathrm{d}_{\mathrm{B}}\right)$. We also define the yellow of the digital input $\mathrm{d}_{Y}$ as $\mathbf{I}\left(d_{Y}, d_{Y}\right.$, $0)$ or $\mathbf{I}_{Y}\left(d_{Y}\right)$ and the gray of the digital input $\mathrm{d}_{\mathrm{k}}$ as $\mathbf{I}\left(d_{K}, d_{K}\right.$, $\left.d_{K}\right)$ or $\mathbf{I}_{K}\left(d_{K}\right)$ in this paper. Using these notations, the XYZ of the arbitrary digital count $\left(\mathrm{d}_{\mathrm{R}}, \mathrm{d}_{\mathrm{G}}, \mathrm{d}_{\mathrm{B}}\right)$ was approximated as follows:

$$
\begin{aligned}
& \hat{\mathbf{I}}\left(d_{R}, d_{G}, d_{B}\right)= \hat{\mathbf{I}}_{K}\left(d_{B}\right)+\left\{\hat{\mathbf{I}}_{Y}\left(d_{G}\right)-\hat{\mathbf{I}}_{Y}\left(d_{B}\right)\right\} \\
&+\left\{\hat{\mathbf{I}}_{R}\left(d_{R}\right)-\hat{\mathbf{I}}_{R}\left(d_{G}\right)\right\}, \\
& \text { if } d_{B}<d_{G}<d_{R},
\end{aligned}
$$

where $\hat{\mathbf{I}}_{i}\left(d_{i}\right)$ denotes the approximation of $\mathbf{I}_{i}\left(d_{i}\right)$, which is explained in the next section. Equation (6) is a more accurate approximation compared to the conventional model that uses only $\hat{\mathbf{I}}_{R}\left(d_{R}\right), \hat{\mathbf{I}}_{G}\left(d_{G}\right), \hat{\mathbf{I}}_{B}\left(d_{B}\right)$ since channel interaction is considered by introducing $\hat{\mathbf{I}}_{K}\left(d_{K}\right), \hat{\mathbf{I}}_{Y}\left(d_{Y}\right)$.
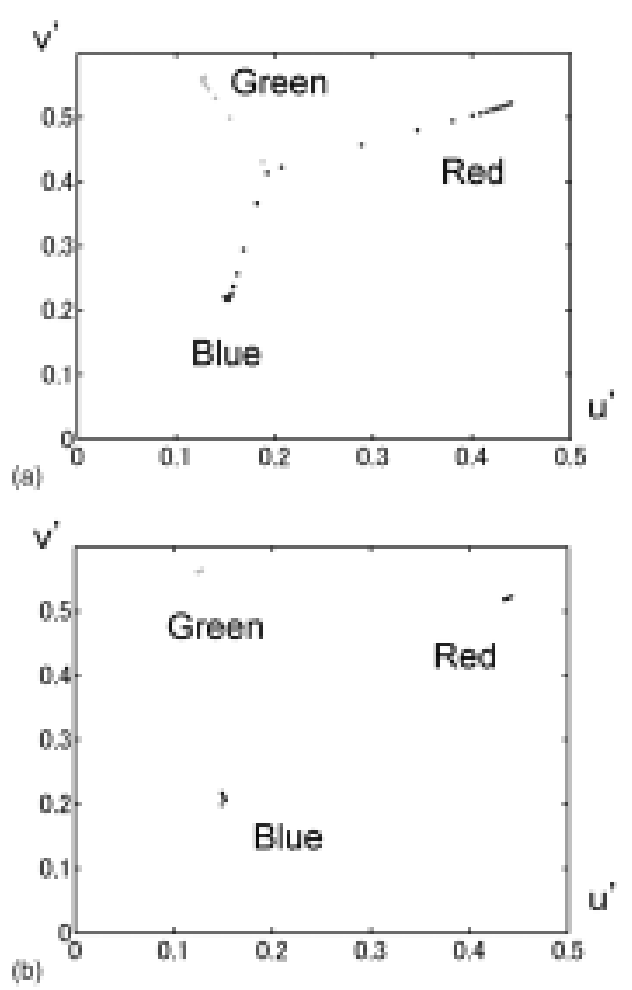

FIGURE 4 - Color tracking before (a) and after (b) black correction.

\subsection{Non-constancy of channel chromaticity}

Figure 4 shows the chromaticity of each primary color where digital input is varied from 0 to 255 with an interval of 15 . From Fig. 4, the channel chromaticity is nearly constant after the subtraction of black (black correction). The subtraction of black is necessary for the LCDs to remove the effect of backlight leakage. In the Masking model, the XYZ of the single channel (R, G, B, C, M, Y, Gr) was calculated as follows.

$\hat{\mathbf{I}}_{i}\left(d_{i}\right)=C_{i}\left(d_{i}\right)\left[\begin{array}{c}X_{i, P C A} \\ Y_{i, P C A} \\ Z_{i, P C A}\end{array}\right]+\mathbf{I}(0,0,0) \quad(i=R, G, B, C, M, Y, G r)$

In order to reduce the error caused by a slight deviation in channel chromaticity, we applied the principal component analysis to the measured XYZ value of each channel. By using PCA, the measured XYZ values are represented by a single vector with minimum error. $\mathrm{X}_{\mathrm{i}, \mathrm{PCA}}, \mathrm{Y}_{\mathrm{i}, \mathrm{PCA}}, \mathrm{Z}_{\mathrm{i}, \mathrm{PCA}}$ in Eq. (7) denotes the first principal component of the measured data and was normalized to be a unit length. $\mathrm{C}_{\mathrm{i}}\left(\mathrm{d}_{\mathrm{i}}\right)$ for the measured digital count $d_{i}$ can be calculated as

$$
C_{i}\left(d_{i}\right)=\left\{\mathbf{I}_{i}\left(d_{i}\right)-\mathbf{I}(0,0,0)\right\}^{T}\left[\begin{array}{c}
X_{i, P C A} \\
Y_{i, P C A} \\
Z_{i, P C A}
\end{array}\right] .
$$

$\mathrm{C}_{\mathrm{i}}$ for an arbitrary digital input can be calculated by interpolating measured $\mathrm{C}_{\mathrm{i}}$ using spline interpolation. In order to 
exclude the device-dependent assumption, no specific function was assumed in this model.

\subsection{Transformation from $\mathrm{XYZ}$ to $\mathrm{RGB}$}

In many practical cases, the conversion from XYZ to corresponding digital inputs is required. From Eqs. (6) and (7), transformation from digital inputs to $\mathrm{XYZ}$ can be written as follows:

$$
\begin{aligned}
{\left[\begin{array}{c}
X \\
Y \\
Z
\end{array}\right]=} & \left\{C_{i}\left(d_{i}\right)-C_{i}\left(d_{j}\right)\right\} \mathbf{P}_{i}+\left\{C_{j}\left(d_{j}\right)-C_{j}\left(d_{G r}\right)\right\} \mathbf{P}_{j} \\
& +C_{G r}\left(d_{K}\right) \mathbf{P}_{G r}+\mathbf{I}(0,0,0),
\end{aligned}
$$

where $i(i=R, G, B)$ and $j(j=C, M, Y)$ are indices of the primary and secondary colors respectively. $\mathbf{P}$ represents vector $\left[\mathrm{X}_{\mathrm{PCA}}, \mathrm{Y}_{\mathrm{PCA}}, \mathrm{Z}_{\mathrm{PCA}}\right]$ in Eq. (7). The $\mathrm{C}_{\mathrm{i}}, \mathrm{C}_{\mathrm{j}}$, and $\mathrm{C}_{\mathrm{Gr}}$ can be obtained using the inverse matrix as follows:

$$
\left[\begin{array}{c}
C_{i}\left(d_{i}\right)-C_{i}\left(d_{j}\right) \\
C_{j}\left(d_{j}\right)-C_{j}\left(d_{G r}\right) \\
C_{G r}\left(d_{G r}\right)
\end{array}\right]=\left[\begin{array}{ll}
\mathbf{P}_{i} \mathbf{P}_{j} \mathbf{P}_{G r}
\end{array}\right]^{-1}\left(\left[\begin{array}{c}
X \\
Y \\
Z
\end{array}\right]-\mathbf{I}(0,0,0)\right) .
$$

From Eq. (10), we can calculate the digital input of gray $\mathrm{d}_{\mathrm{Gr}}$ using $\mathrm{C}_{\mathrm{Gr}}$ defined in Eq. (8) using spline interpolations. It is known that spline interpolation is a very effective interpolation method for any smooth curve. Similarly, $d_{i}$ and $\mathrm{d}_{\mathrm{j}}$ can be calculated using $\mathrm{d}_{\mathrm{K}}$ and $\mathrm{C}_{\mathrm{i}}, \mathrm{C}_{\mathrm{j}}$. There are six possibilities of $(\mathrm{i}, \mathrm{j}) ;(\mathrm{R}, \mathrm{M}),(\mathrm{R}, \mathrm{Y}),(\mathrm{G}, \mathrm{Y}),(\mathrm{G}, \mathrm{C}),(\mathrm{B}, \mathrm{C})$, and $(\mathrm{B}, \mathrm{M})$. We select the combinations which satisfy following conditions. Conditions defined in Eq. (11) represents the physical relevancy since digital counts range from 0 to 255 for an 8-bit display system.

$$
\left\{\begin{array}{l}
d_{K} \leq d_{j} \leq d_{i} \\
0 \leq d_{K}, d_{j}, d_{i} \leq 255
\end{array}\right.
$$

\section{$4 \quad$ Modified masking model}

The circles shown in Fig. 5 are the tracking of the XYZ values in the $\mathrm{R}$ channel. From Fig. 5, it is clear that the approximation using Eq. (7) is not effective since the deviation from linearity is very large. The inclusion of a higherorder principal component is a common method for principal component analysis when the error is large for only one principal component. Therefore, we add the higher-order principal component vector to approximate the curve-shaped tracking. For the case in Fig. 5, the second principal component is a vector where the direction is almost the same as the direction of the $\mathrm{Z}$ axis. Figure 6 shows the relationship between digital input $\mathrm{d}_{\mathrm{R}}$ and the coefficient of the second principal component. From Fig. 6, it can be seen that the second principal component has a significant effect, since its peak magnitude is close to 2 . For

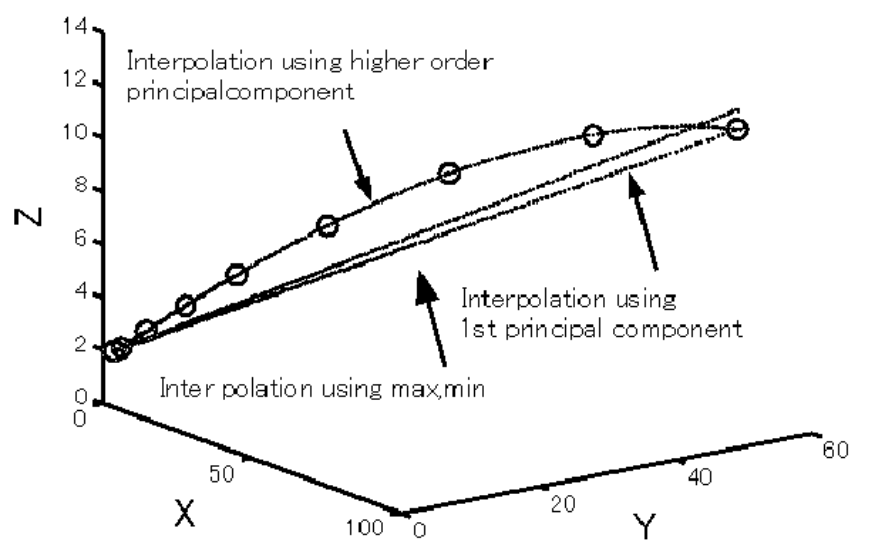

FIGURE 5 - Tracking of XYZ in R channel (Sony PCG-C1MR/BP).

the Modified Masking model, the XYZ of the single channel is calculated by using Eq. (12) instead of Eq. (7):

$\hat{\mathbf{I}}_{i}\left(d_{i}\right)=\sum_{j=1}^{2} C_{i j}\left(d_{i}\right)\left[\begin{array}{c}X_{i, P C A} \\ Y_{i, P C A} \\ Z_{i, P C A}\end{array}\right]+\mathbf{I}(0,0,0) \quad(i=R, G, B, C, M, Y, G r)$.

In Eq. (12), index PCAj represents the j-th component of PCA. $\mathrm{C}_{\mathrm{ij}}$ is calculated by using Eq. (13):

$$
C_{i j}\left(d_{i}\right)=\left\{\mathbf{I}_{i}\left(d_{i}\right)-\mathbf{I}(0,0,0)\right\}^{T}\left[\begin{array}{c}
X_{i, P C A j} \\
Y_{i, P C A j} \\
Z_{i, P C A j}
\end{array}\right] .
$$

Figure 5 shows that the prediction based on Eqs. (1), (7), and (12) is improved by considering a higher-order principal component.

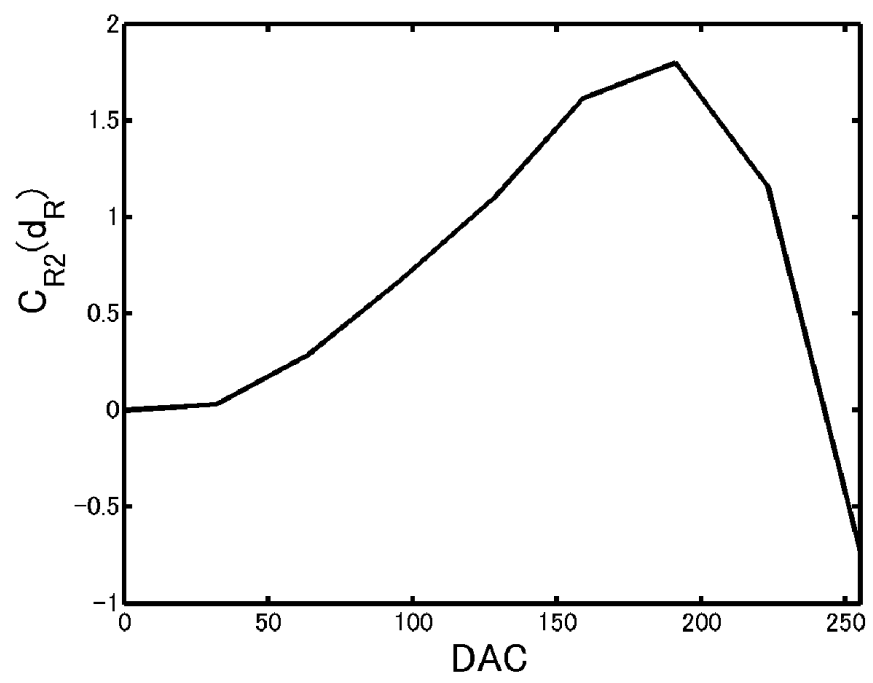

FIGURE 6 - Coefficient of the second principal component (Sony PCG-C1MR/BP). 


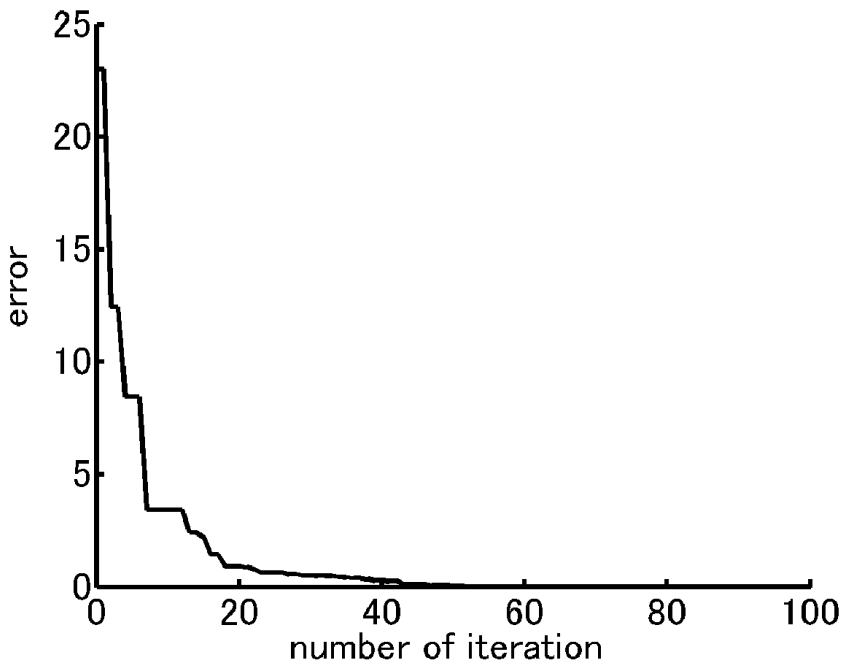

FIGURE 7 - Error vs number of iterations.

\subsection{Transformation from $X Y Z$ to $R G B$}

For the Modified Masking model, the conversion from XYZ to corresponding digital inputs is rather complicated compared to that of the Masking model. Corresponding digital inputs $\mathrm{d}_{\mathrm{R}}, \mathrm{d}_{\mathrm{G}}$, and $\mathrm{d}_{\mathrm{B}}$ were calculated by minimizing the square error defined as follows:

$$
\text { error }=\left\|\left[\begin{array}{c}
X \\
Y \\
Z
\end{array}\right]-\hat{\mathbf{I}}\left(d_{R}, d_{G}, d_{B}\right)\right\| .
$$

Figure 7 shows the relationship between error and the number of iterations when XYZ is set to $(140,140,140)$ to see the convergence for the white case. The Nelder-Mead method was used for optimization. The initial value of the digital inputs was calculated by using Eqs. (9), (10), and (11). From Fig. 7, it is clear that the error converges smoothly. In general, the initial RGB value was calculated by using Eqs. (9), (10), and (11) which is not far from the converged RGB value. Therefore, the error is converges smoothly due to the characteristics of the simplex method.

\section{Comparison of model performance}

The performance of the Masking model and the Modified Masking model was tested and compared with conventional characterization methods; GOG, S-curve, Polynomial, and Matrix models. Although the GOG model was originally designed for the characterization of CRTs, comparison with the Masking model was performed since it is also used for LCDs in some other papers (such as Ref. 4).

\subsection{Condition of measurement}

Table 1 shows three type of LCDs used in this experiment. The generated colors were displayed on a full screen. The
TABLE 1 - Lists of measured displays.

\begin{tabular}{cccc}
\hline \hline & Model & Type & Resolutions \\
\hline LCD1 & SHARP & 18.1-in. TFT-LCD & \\
& LL-TI80A & monitor & $1280 \times 1024$ \\
LCD2 & iiyama & 18.1-in. a-Si TFT- & \\
& AS4635U & LCD monitor & $1280 \times 1024$ \\
LCD3 & Sony & 8.9-in. Ultra-Wide & \\
& PCG-C1MR/BP & TFT-LCD (Laptop) & $1280 \times 600$ \\
\hline \hline
\end{tabular}

spectral radiance of the LCD was measured at a $2^{\circ}$ field of central area using a spectroradiometer (MINOLTA CS1000). The distance of the spectroradiometer from the LCD screen was $30 \mathrm{~cm}$. The colors were displayed in the entire screen.

\subsection{Channel interaction}

Table 2 shows the characteristics of the channel interaction for the measured LCDs. The error caused by the channel interaction was calculated by subtracting the third column from the second column. It is clear that channel interaction is very large except for LCD2.

\subsection{Measurement of training data}

For the Matrix model, we measured eight color scales from 8 to 255 with equal steps per channel and 32 neutral colors (see Ref. 3). We used the same digital inputs as defined in Ref. 3. For the GOG, Polynomial, and S-curve models, the measured data contains XYZ of equally spaced 5, 9, 18, and 32 steps from 0 to 255 per channel. The XYZ of four different steps were measured to check the dependency on the amount of training data. The parameter for each model was calculated to minimize the error between estimation and training data. Finally, the training data for the Masking model and Modified Masking model contains equally spaced 5, 9, 18, and 32 steps for R, G, B, C, M, Y, and Gray.

\subsection{Result and discussion}

One-hundred colors were randomly generated and measured as test data to evaluate the performance of each model. The difference between the XYZ of measured test data and predicted XYZ was evaluated using the CIE94 color difference. Table 3 shows the average color difference for each model. Figure 8 shows the relationship between the average color difference and the number of training data for each

TABLE 2 - Results of channel interaction test.

\begin{tabular}{cccc}
\hline \hline & $\mathbf{I}(140,140,140)$ & $\begin{array}{c}\mathbf{I}_{\mathbf{R}}(140)+\mathbf{I}_{\mathbf{G}}(140)+ \\
\mathbf{I}_{\mathbf{B}}(140)-2 \mathbf{I}_{\mathbf{0}} \times Y Z\end{array}$ & $\begin{array}{c}\text { Error } \\
\Delta X \Delta Y \Delta Z\end{array}$ \\
\hline LCD1 & $32.3,33.0,35.1$ & $22.1,22.5,24.2$ & $10.2,10.5,10.9$ \\
LCD2 & $30.1,31.9,23.1$ & $29.6,31.4,22.6$ & $0.5,0.5,0.5$ \\
LCD3 & $46.7,46.4,66.9$ & $52.3,52.1,74.2$ & $-5.6,-5.7,-7.3$ \\
\hline \hline
\end{tabular}



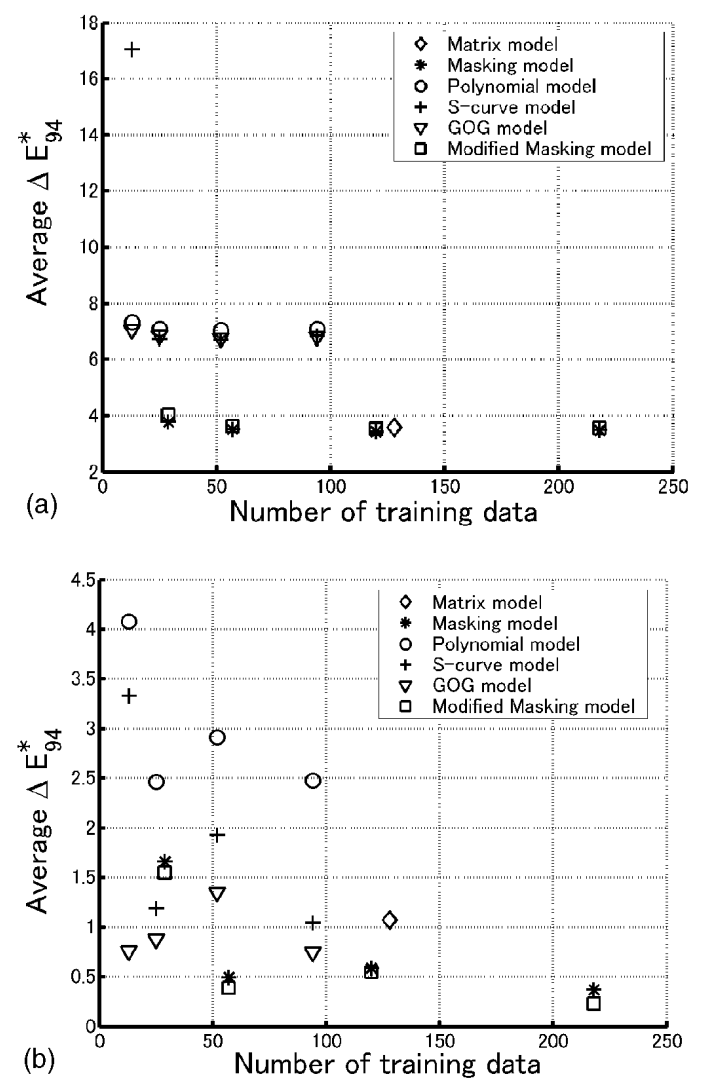

(b)

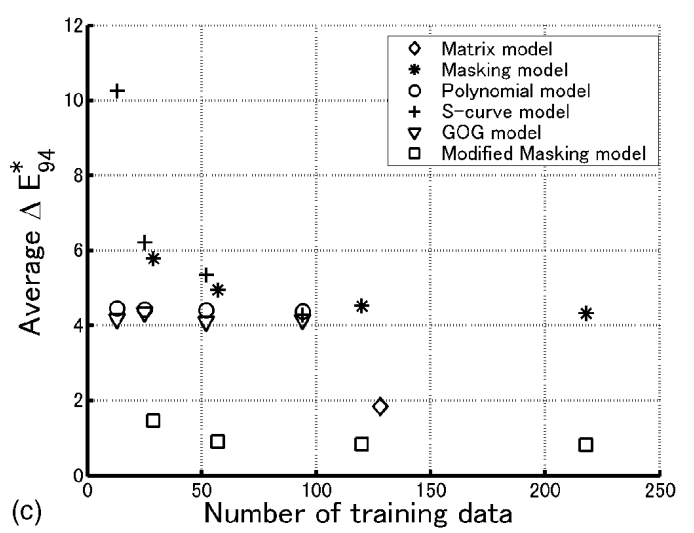

FIGURE 8 - Amount of training data vs. average $\Delta E_{94} *$ : (a) LCD1, (b) LCD2, (c) LCD3.

display. From Fig. 8, we can see the improvement by increasing the training data. The GOG model is more accurate than either the S-curve model or the Polynomial model. This is due to the correction of the transfer function by manufacturers to approximate the gamma curve. For the S-curve model, the larger prediction error in for the small number of training data can be seen in the figures. It is considered as a shortage of training data since the S-curve model has many parameters that need to be calculated from training data. Predictions by the Matrix model is quite accurate for all types of LCDs. The Masking model is more accurate than almost all of the characterization methods. However, the accuracy of prediction is decreased for LCD3. This is due to the nonlinearity of chromaticity change as
TABLE 3 - Performance results of the characterization model.

\begin{tabular}{|l|c|c|c|c|}
\hline \multicolumn{2}{|c|}{ Average $\Delta E_{94}^{*}$} & \multirow{2}{*}{ LCD1 } & LCD2 & LCD3 \\
\hline \multirow{3}{*}{$\begin{array}{l}\text { Modified } \\
\text { Masking }\end{array}$} & 5 step & 4.04 & 1.55 & 1.46 \\
\cline { 2 - 5 } & 9 step & 3.63 & 0.39 & 0.91 \\
\cline { 2 - 5 } & 18 step & 3.53 & 0.55 & 0.84 \\
\cline { 2 - 5 } & 32 step & 3.56 & 0.23 & 0.82 \\
\hline \multirow{4}{*}{$\begin{array}{l}\text { Masking } \\
\text { Model }\end{array}$} & 5 step & 3.77 & 1.66 & 5.79 \\
\cline { 2 - 5 } & 9 step & 3.52 & 0.50 & 4.95 \\
\cline { 2 - 5 } & 18 step & 3.43 & 0.59 & 4.52 \\
\cline { 2 - 5 } & 32 step & 3.49 & 0.37 & 4.33 \\
\hline \multirow{4}{*}{$\begin{array}{l}\text { S-curve } \\
\text { Model }\end{array}$} & 5 step & 17.05 & 3.33 & 10.26 \\
\cline { 2 - 5 } & 9 step & 6.73 & 1.19 & 6.22 \\
\cline { 2 - 5 } & 18 step & 6.70 & 1.93 & 5.35 \\
\cline { 2 - 5 } & 32 step & 6.86 & 1.05 & 4.29 \\
\hline \multirow{3}{*}{$\begin{array}{l}\text { Polynomial } \\
\text { Model }\end{array}$} & 5 step & 7.34 & 4.08 & 4.45 \\
\cline { 2 - 5 } & 9 step & 7.10 & 2.47 & 4.43 \\
\cline { 2 - 5 } & 18 step & 7.05 & 2.91 & 4.40 \\
\cline { 2 - 5 } & 32 step & 7.10 & 2.48 & 4.38 \\
\hline \multirow{3}{*}{$\begin{array}{l}\text { GOG } \\
\text { Model }\end{array}$} & 5 step & 7.05 & 0.76 & 4.16 \\
\cline { 2 - 5 } & 9 step & 6.86 & 0.87 & 4.35 \\
\cline { 2 - 5 } & 18 step & 6.76 & 1.35 & 4.11 \\
\cline { 2 - 5 } & 32 step & 6.81 & 0.75 & 4.16 \\
\hline Matrix model & & 3.53 & 1.97 & 1.84 \\
\hline
\end{tabular}

shown in Fig. 5. The Modified Masking model outperformed all conventional models. For the case of LCD2, all the characterization models are accurate because LCD2 has less channel interaction as shown in Table 2.

\section{Conclusion}

An accurate colorimetric characterization method for LCDs based on the Masking model is introduced. The Masking model takes into consideration the channel interaction and non-constancy of the primaries. A performance comparison with conventional characterization methods proved that the proposed method is very effective in the colorimetry of LCDs. Experimental results show that the number of measurements required to calculate the model parameters is much less than for conventional models. Model parameters are easily calculated by using PCA and spline interpolation because the computational load for these method is very small. From the experimental results of our research, the inclusion of channel interaction characteristics to the ICC profile would result in more accurate color reproduction.

\section{References}

1 R S Berns, R J Motta, and M E Gorzynski, "CRT Colorimety,” COLOR research and application 18, No. 5, 299-314 (October 1993).

2 Y Kwak and L W MacDonald "Accurate Prediction of Colours on Liquid Crystal Displays,” Proc ISむT/SID Ninth Color Imaging Conf, 355-359 (November 2001).

3 IEC 61966-4: "Multimedia system and equipment: Colour measurement and management. Part 4: Equipment Using Liquid Crystal Display Panels." 
4 J E Gibson and M D Fairchild, "Colorimetric Characterization of Three Computer Displays," Munsell Color Sci Lab Tech Report (2000).

5 M D Farichild and D R Wyble, "Colorimetric Characterization of the Apple Studio Display,” Munsell Color Sci Lab Tech Report (1998).

6 N Tamura, T Ishii, N Tsumura, Y Yoshida, Y Yamamoto, and Y Miyake, "Evaluation of image quality on reflective-type LCD: Calibration Based on PCA," Proc IS\&T 2001 PICS Conf, 368-371 (2001).

7 N Tamura, T Ishii, N Tsumura, Y Yoshida, Y Yamamoto, and Y Miyake, "Calibration of LCD Colorimetry Based on Principal Component Analysis," Proc Asia Display/IDW ‘01, 1545-1548 (2001).

8 Y Yoshida and Y Yamamoto, "Color Calibration of LCDs," Proc IS\&T/SID Tenth Color Imaging Conf, 305-311 (November 2002).

9 Y Miyake, H Saitoh, H Yaguchi, and N Tsukada "Facial Pattern Detection and Color Correction from Television Picture for Newspaper Printing," J Imag Technol 16, 165-169 (October 1990).

$10 \mathrm{~J}$ A N lder and R Mead, "A Simplex Method for Function Minimization," Comput J 7, 308-313 (1965).

$11 \mathrm{~J}$ A C Yule, Principles of Color Reproduction (John Wiley \& Sons, 1967).

12 W K Pratt, Digital Image Processing (John Wiley \& Sons, 1978).

Nobuhiko Tamura received his B.E. and M.E. degrees from the Department of Information and Computer Science from Chiba University in 2000 and 2002 respectively. He received the first prize for his master's thesis in the department. Now, he is the doctorate course student at Chiba University. He is interested in multispectral imaging, medical imaging, and virtual reality. He is a member of Japanese Society for Medical Virtual Reality.
Norimichi Tsumura received his B.E., M.E., and Dr. Eng degrees in applied physics from Osaka University in 1990, 1992, and 1995, respectively. He moved to the Department of Information and Computer Sciences, Chiba University, in April 1995, as an assistant professor. He was a visiting scientist in the University of Rochester from March 1999 to January 2000. He is currently an associate professor at the Department of Information and Image Sciences, Chiba University. He is also a researcher at PREST, Japan Science and Technology Corporation (JST).

Professor Miyake has been professor in the Department of Information and Image Sciences, Chiba University, since 1989. He received his Ph.D. from the Tokyo Institute of Technology in 1978 for his study of color image processing and analysis. He received many awards from the SPST) (Society of Photographic Science and Technology of Japan) and others. For example, he received the Charles E. Ives award in 1991 and 2002 from IS\&T; he became a Fellow of IS\&T in 1995. In January 2000, IS\&T and SPIE named him the 2000 Electronic Imaging Honoree of the Year. From 1990 to 1993 he served as editor in chief of the Journal of SPSTJ. He served as president of SPSTJ from 1999 to 2001 . He is currently vice president of IS\&T. He has published more than 200 original papers and 17 books in the field of color image processing, analysis, and evaluation. He is a one of the pioneers of the multi-spectral imaging researches. 